\title{
Carotid Plaque Characterization Using 3D T1-Weighted MR Imaging with Histopathologic Validation: A Comparison with 2D Technique
}

\author{
S. Narumi, M. Sasaki, T. Natori, M. Yamaguchi Oura, K. Ogasawara, M. Kobayashi, Y. Sato, Y. Ogasawara, J. Hitomi, and Y. Terayama
}

\begin{abstract}
BACKGROUND AND PURPOSE: 3D FSE TIWI has recently been used for carotid plaque imaging, given the potential advantages in contrast and spatial resolutions. However, its diagnostic performance remains unclear. Hence, we compared the ability of this technique to readily assess plaque characteristics with that of conventional images and validated the results with histologic classification.
\end{abstract}

MATERIALS AND METHODS: We prospectively examined 34 patients with carotid stenosis who underwent carotid endarterectomy by using 1.5T scanners and obtained 3D-FSE TIWI and 2D spin-echo TIWI scans. After generating reformatted images obtained from the 3D-FSE T1-weighted images, we calculated the contrast ratios for the plaques and the adjacent muscles and compared these findings with the pathologic classifications.

RESULTS: Carotid plaques were histologically classified as types VII, VIII, IV-V, or VI. With 3D-FSE TIWI, the range of contrast ratios for each classification was the following: 0.94-0.97 (median, 0.95), 0.95-1.29 (median, 1.10), 1.33-1.54 (median, 1.42), and 1.53-2.12 (median, 1.80), respectively. With 2D imaging, the range of contrast ratios for each classification was the following: 0.79-1.02 (median, 0.90), $0.88-1.19$ (median, 1.01), 1.17-1.46 (median, 1.23), and 1.55-2.51 (median, 2.07), respectively. Results were significantly different among the 4 groups $(P<$ .001 ). Sensitivity and specificity for discriminating vulnerable plaques (IV-VI) from stable plaques (VII, VIII) were both 100\% for the 3D technique and $100 \%$ and $91 \%$, respectively, for the 2D technique.

CONCLUSIONS: 3D-FSE TIWI accurately characterizes intraplaque components of the carotid artery, with excellent sensitivity and specificity compared with those of 2D-TIWI.

ABBREVIATIONS: $\mathrm{CR}=$ contrast ratio; $\mathrm{SE}=$ spin-echo

C ervical carotid stenosis is an important cause of cerebral infarction and transient ischemic attack. Carotid endarterectomy or carotid artery stent placement is performed to prevent future stroke events but may also cause embolic complications during the surgery, especially if the plaque contains substantial vulnerable components such as intraplaque hemorrhage or lipid. ${ }^{1,2}$ Therefore, establishing a method for characterizing intraplaque components is an important prerequisite for predicting perisurgical complications.

\footnotetext{
Received August 4, 2014; accepted after revision October 2.

From the Departments of Neurology and Gerontology (S.N., T.N., M.Y.O., Y.T.), Neurosurgery (K.O., M.K., Y.S., Y.O.), and Anatomy (J.H.), and Institute for Biomedical Sciences (M.S.), Iwate Medical University, Morioka, Japan.

This work was partly supported by Grants-in-Aid for Strategic Medical Science Research (S1491001, 2014-2018) and for Scientific Research (25861122) from the Ministry of Education, Culture, Sports, Science, and Technology of Japan.

Please address correspondence to Shinsuke Narumi, MD, PhD, Department of Neurology, Iwate Medical University, 19-1 Uchimaru, Morioka 020-8505, Japan; e-mail: snarumi@iwate-med.ac.jp

- Indicates open access to non-subscribers at www.ajnr.org

http://dx.doi.org/10.3174/ajnr.A4197
}

Several modalities have been used for plaque characterization, including ultrasonography and MR imaging. Although ultrasonography is widely used, the interpretation is typically subjective and may be impossible in the presence of extensive calcification or a high-positioned carotid bifurcation. Although gray-scale median and integrated backscatter have been introduced as quantitative metrics, previous reports suggest that they are unsuitable for evaluating intraplaque components. ${ }^{3,4} \mathrm{MR}$ plaque imaging is another popular method for assessing plaque characteristics. Although various imaging techniques have been used, a 2D spinecho (SE) T1WI technique with appropriate scanning parameters has been reported to accurately quantify intraplaque components, compared with other conventional techniques. ${ }^{5-8}$ Recently, a 3D T1WI FSE technique has been adopted for this purpose because it can minimize partial volume effects and motion artifacts, as well as enhance black-blood effects, while maintaining T1WI contrast. However, whether the 3D-FSE technique can more accurately discriminate among intraplaque components than the more conventional techniques, such as 2D-SE T1WI, 
Table 1: Histologic classification of plaque specimens excised by carotid endarterectomy

\begin{tabular}{llcc}
\hline Type $^{\text {a }}$ & \multicolumn{1}{c}{ Description } & No. (\%) & [Symptomatic/Asymptomatic] \\
\hline I-II & Near-normal wall thickness without calcification & $0(0 \%)$ & \\
III & Diffuse intimal thickening or small eccentric plaque without calcification & $0(0 \%)$ & $6(19 \%)$ \\
IV-V & Lipid-rich or necrotic core surrounded by fibrous tissue with possible calcification & $14(45 \%)$ & {$[5 / 1]$} \\
VI & Complex plaque with possible surface defect, hemorrhage, or thrombus & $2(7 \%)$ & {$[14 / 0]$} \\
VII & Calcified lesion & $9(29 \%)$ & {$[1 / 1]$} \\
VIII & Fibrotic plaque without a lipid core and with possible small calcifications & {$[5 / 4]$} \\
\hline
\end{tabular}

${ }^{a}$ Modified American Heart Association classification.

remains unknown. Hence, in the present study, we investigated whether the diagnostic accuracy of 3D-FSE T1WI, in terms of carotid plaque characterization, is comparable with that of $2 \mathrm{D}-\mathrm{SE}$ T1WI, by using pathologic specimens excised from carotid endarterectomy as our validation standards.

\section{MATERIALS AND METHODS \\ Patients}

From July 2012 to December 2013, we prospectively examined 34 consecutive patients (age range, 58-79 years [mean, 70.4 years]; 31 men and 3 women) with substantial stenosis of the cervical internal carotid artery, all of whom underwent carotid endarterectomy. Of these patients, 26 had symptomatic stenosis of 50\%95\%, according to the method of the North American Symptomatic Carotid Endarterectomy Trial, ${ }^{9}$ and 8 had asymptomatic stenosis of $70 \%-90 \%$. The clinical profiles of the patients included hypertension $(n=28)$, hyperlipidemia $(n=27)$, and diabetes mellitus $(n=11)$. The institutional review board approved the study protocol, and written informed consent was obtained from all patients.

\section{Imaging Protocol}

Presurgery sagittal 3D-T1WI of the bilateral carotid bifurcation was performed by using a 1.5T MR imaging scanner (Signa HDxt; GE Healthcare, Milwaukee, Wisconsin) with an 8-channel neurovascular coil. The pulse sequence parameters were as follows: flow-sensitized 3D-FSE with variable flip angles; TR/TE, 500/18.3 ms; echo-train length, 24; b-value of the flow-sensitized gradients along 3 axes, $2.2 \mathrm{~s} / \mathrm{mm}^{2}$; FOV , $25 \times 19 \mathrm{~cm}^{2}$; matrix size, $512 \times 512$ (after zero-fill interpolation); section interval, $0.5 \mathrm{~mm}$ (after zerofill interpolation); partitions, 248 ; voxel size, $0.5 \times 0.5 \times 0.5 \mathrm{~mm}^{3}$; parallel imaging factor, 2; NEX, 1; fat suppression, chemical shift selective suppression; and acquisition time, 3 minutes 54 seconds. Motion-sensitized gradients of $2.2 \mathrm{~s} / \mathrm{mm}^{2}$ were used as the blackblood technique.

In addition, axial 2D-T1WI of the affected carotid bifurcation was performed by using a 1.5T MR imaging scanner (Echelon Vega; Hitachi Medical Corporation, Tokyo, Japan) with an 8-channel neurovascular coil. The section direction was carefully set in a plane perpendicular to the long axis of the carotid bifurcation by using sagittal $2 \mathrm{D}$ phase-contrast MR angiographic imaging. The pulse sequence parameters were as follows: conventional SE with a radial $k$-space acquisition technique with self-navigation, which is similar to the periodically rotated overlapping parallel lines with enhanced reconstruction method ${ }^{10}$; TR/TE, 500/12 ms; FOV, $18 \mathrm{~cm}$; matrix size, $512 \times 512$ (after zero-fill interpolation); NEX, 2; section thickness, $4.0 \mathrm{~mm}$ with intersection gaps of $1.0 \mathrm{~mm}$; number of sections, 9; fat suppression, chemical shift selective suppression; and acquisition time, 6 minutes 46 seconds. Nonselective saturation pulses were used as the black-blood technique.

\section{Histologic Preparation}

Specimens were excised en bloc from the affected carotid arteries and submitted for histologic evaluation. The specimens were fixed in formaldehyde, and transverse sections of the carotid bifurcations were carefully cut to correspond in direction and position to the 2D-MR images. During histologic preparation, hematoxylin-eosin, Masson trichrome, and anti-Glycophorin-A stains were applied to paraffin-embedded, $7-\mu \mathrm{m}$-thick sections. The sections of carotid plaque were classified into 6 types, according to the modified American Heart Association classification (Table 1). ${ }^{11}$

\section{Data Processing and Statistical Analyses}

After using the 3D-FSE images to generate reformatted axial images that corresponded to the 2D-SE images, we measured the signal intensity of the carotid plaque and the adjacent sternomastoid muscle by using the image with the largest plaque size. The images were randomized, and a blinded author (S.N.) manually traced the plaque and muscle 3 times, by using the polygon cursor of a free software package (zioTerm 2009; Ziosoft, Tokyo, Japan). The resulting signal intensity values were averaged, and the contrast ratio (CR) was calculated by dividing the average signal intensity of the plaque by that of the muscle.

Differences in the CRs of plaques among the various histologic types were examined by using the Kruskal-Wallis test, followed by the post hoc Mann-Whitney test. Differences between the 3D-FSE and 2D-SE techniques and those between the asymptomatic and symptomatic patients were examined by using the Wilcoxon signed rank test and the Mann-Whitney test, respectively. In addition, receiver operating characteristic curve analyses were performed to evaluate the sensitivity and specificity of the ability of the techniques to differentiate plaques containing substantial vulnerable components from those containing stable components. Vulnerable components were defined as lipid, necrosis, and/or hemorrhage (IV-V, VI), while stable components were defined as calcification and fibrous tissue (I-II, III, VII, VIII). Receiver operating characteristic curve analysis was also performed to evaluate the abilities of the techniques to differentiate hemorrhagic complex plaques (VI) from other plaques. Cutoff values were determined by using the Youden index, and differences in the areas under the receiver operating characteristic curves were examined by using the DeLong test. The intraclass correlation coefficient was used to evaluate intraoperator agreements for the manual measurements. The $\alpha$ level used was .05. 
Table 2: Contrast ratios of various carotid plaques on T1-weighted images using 3D FSE and 2D-SE techniques ${ }^{\mathrm{a}}$

\begin{tabular}{|c|c|c|c|c|c|}
\hline & \multicolumn{4}{|c|}{ Plaque Type (Modified AHA Classification) } & \multirow[b]{2}{*}{$P$ Value $^{\mathrm{b}}$} \\
\hline & VII $(n=2)$ & VIII $(n=9)$ & $\mathrm{IV}-\mathrm{V}(n=6)$ & VI $(n=14)$ & \\
\hline 3D-FSE & $0.94-0.97(0.95)$ & $0.95-1.29(1.10)$ & $1.33-1.54$ (1.42) & $1.53-2.12(1.80)$ & $<.001$ \\
\hline 2D-SE & $0.79-1.02(0.90)$ & $0.88-1.19$ (1.01) & $1.17-1.46(1.23)$ & $1.55-2.51(2.07)$ & $<.001$ \\
\hline$P$ value ${ }^{c}$ & .655 & .139 & .028 & .013 & \\
\hline
\end{tabular}

Note:-AHA indicates American Heart Association.

${ }^{a}$ Contrast ratios are presented as range (median).

${ }^{\mathrm{b}}$ Kruskal-Wallis test.

c Wilcoxon signed rank test.

3D-FSE T1W images

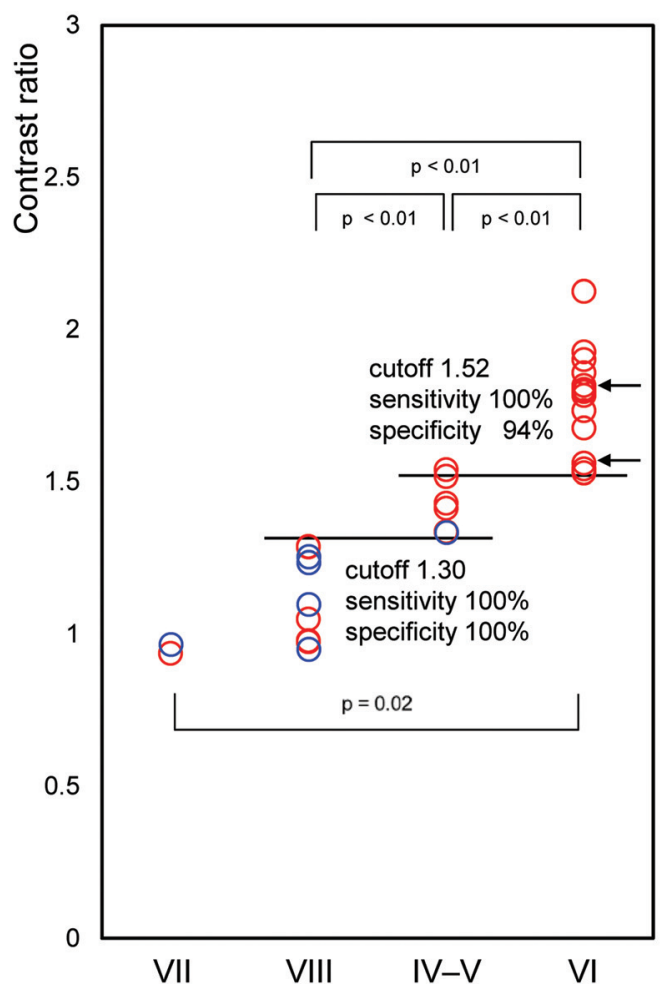

\section{D-SE T1W images}

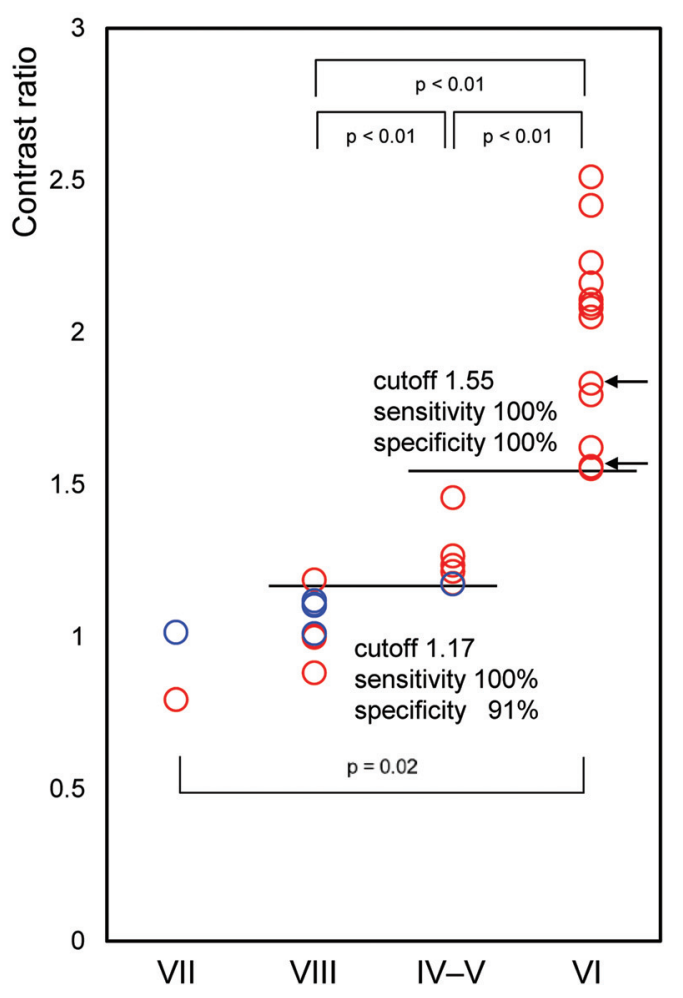

FIG 1. Contrast ratios of carotid plaques with various compositions, by using 3D-FSE and 2D-SE TIWI. Contrast ratios of type VI plaques (characterized according to the modified American Heart Association criteria) are significantly higher than those of other plaque types, by using both 3D-FSE and 2D-SE images. In addition, CRs of type IV-V plaques are significantly higher than those of types VII and VIII and are significantly higher on 3D-FSE images than on 2D-FSE images. Sensitivity and specificity for discriminating between vulnerable plaques (IV-V and VI) and stable plaques (VII and VIII) were both 100\%, by using the 3D-FSE images, and 100\% and 91\%, respectively, by using the 2D-SE images. Red circles indicate symptomatic patients; blue circles, asymptomatic patients; and arrows, patients showing ischemic events during surgery.

\section{RESULTS}

Three patients were excluded because their carotid endarterectomy was postponed for $>3$ months after their MR imaging examinations. The remaining 31 patients were considered eligible for further quantitative analyses. Patient characteristics are as follows: age, ranging from 58 to 79 years (mean, 70.8 years); 28 men and 3 women; and interval between MR imaging and carotid endarterectomy, ranging from 1 to 82 days (median, 28 days). The number and type of carotid plaques, as determined by histopathologic examination, are shown in Table 1. Ischemic stroke events during the surgery occurred in the 2 symptomatic patients with carotid plaque type VI.

With 3D-FSE T1WI, the CR range and median values of the plaques that were histologically confirmed as types VII, VIII, IV-V, and VI were 0.94-0.97 (0.95), 0.95-1.29 (1.10), 1.33-1.54 (1.42), and 1.53-2.12 (1.80), respectively. The CR range and median value by using 2D-SE T1WI were 0.79-1.02 (0.90), $0.88-$ 1.19 (1.01), 1.17-1.46 (1.23), and 1.55-2.51 (2.07), respectively.
These values were significantly different among the groups, by using both the 3D-FSE and 2D-SE images $(P<.001$, KruskalWallis test; Table 2 and Fig 1). Among the CRs of carotid plaques with various compositions, there were significant differences among all combinations of types VIII, IV-V, and VI, which tended to show isointensity to the muscle, mild hyperintensity, and marked hyperintensity, respectively, on both the 3D-FSE and 2D-SE images $(P<.01$, post hoc Mann-Whitney test; Figs 1 and $2)$. The CRs of types IV-V plaques on the $3 \mathrm{D}$ images were significantly higher than those on the $2 \mathrm{D}$ images $(P=.028$, Wilcoxon signed rank test), while the CRs of the type VI plaques on the $3 \mathrm{D}$ images were significantly lower than those on the $2 \mathrm{D}$ images $(P=$ .013, Wilcoxon signed rank test; Table 1). Furthermore, the CRs of the plaques in the symptomatic patients were significantly higher than those in the asymptomatic patients both on the $3 \mathrm{D}$ images (0.94-2.12 [median, 1.54] and 0.95-1.33 [median, 1.17], respectively; $P=.004$, Mann-Whitney test) and on the $2 \mathrm{D}$ images 

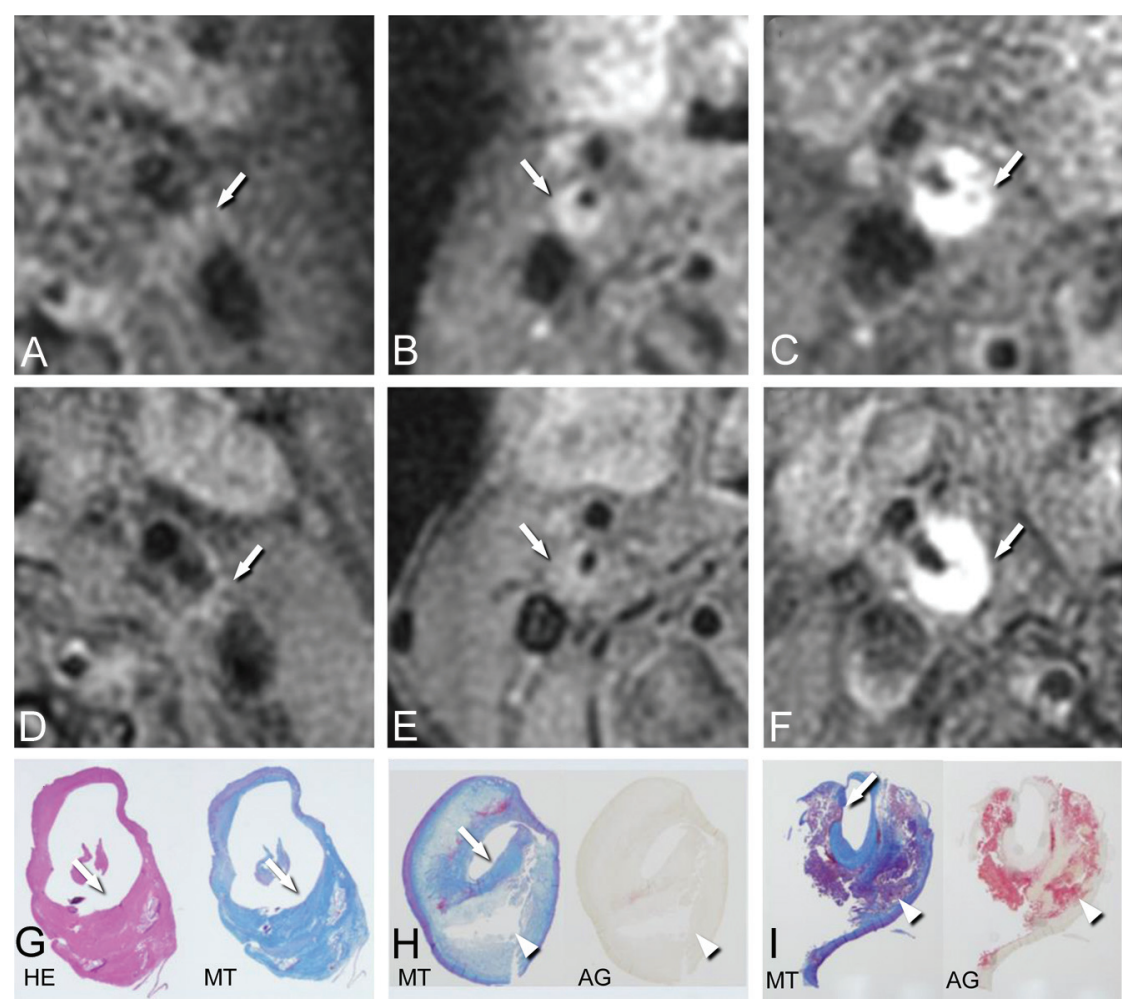

FIG 2. Imaging findings of the carotid plaques by using 3D-FSE and 2D-SE TWI. A-C, 3D-FSE TTWI. D-F, 2D-SE TWW. G-I, Histologic specimens. AG indicates anti-Glycophorin-A staining; HE, hematoxylin-eosin staining; MT, Masson trichrome staining. $G$ and $H, 2 \times$ magnification; I, $1.5 \times$ magnification.

A 74-year-old man with symptomatic left carotid stenosis ( $A, D$, and $G)$. The carotid plaque is isointense to the adjacent muscles on both 3D-FSE and 2D-SE TIWI (arrows, $A$ and D), with CRs of 0.97 and 1.00, respectively. Histologic findings show that the plaque was primarily composed of fibrous tissue with minimal calcification, which is therefore classified as type VIII (arrows, G).

A 69-year-old man with symptomatic right carotid stenosis $(B, E$, and $H$ ). The plaque shows moderate hyperintensity on the 3D-FSE image (arrow, $B$ ) and only subtle hyperintensity on the 2D-SE image (arrow, E), with CRs of 1.52 and 1.27, respectively. Histologic findings show a lipidrich plaque consisting of foamy cells (arrowheads, $H$ ) with a thick fibrous cap (arrow, $H$ ), which is therefore classified as types IV-V.

A 72-year-old man with symptomatic right carotid stenosis $(C, F$, and $I)$. The plaque shows evident hyperintensity on both the 3D-FSE and 2D-SE images (arrows, $C$ and F), with CRs of 1.93 and 2.23, respectively. Histologic findings show massive hemorrhage (arrowheads, I) with a partially ruptured thin fibrous cap (arrow, I), which is therefore classified as type VI.

(0.79-2.51 [median, 1.56] and 1.01-1.17 [median, 1.11], respectively; $P=.023$, Mann-Whitney test).

Receiver operating characteristic analyses revealed that the areas under the receiver operating characteristic curves for the 3DFSE images were 1.000 (95\% CI, 0.888-1.000), and the areas under the receiver operating characteristic curves for the 2D-SE images were 0.991 (95\% CI, 0.871-1.000), indicating that both could discriminate between vulnerable plaques (types IV-V and VI) and stable plaques (types VII and VIII). No significant difference was detected between the 2 techniques $(P=.41$, DeLong test). The sensitivities and specificities were both $100 \%$ for the 3D images (cutoff value, 1.30) and $100 \%$ and $91 \%$, respectively, for the $2 \mathrm{D}$ images (cutoff value, 1.17; Fig 1). Furthermore, in discriminating complex plaques (type VI) from other plaques, the areas under the receiver operating characteristic curves for the $3 \mathrm{D}$ and 2D images were 0.996 (95\% CI, 0.880-1.000) and 1.00 (95\% CI, $0.888-1.000)$, respectively, with no significant difference between the 2 techniques ( $P=.48$, DeLong test). The sensitivities and specificities were $100 \%$ and $94 \%$, respectively, for the 3D images (cutoff value, 1.52), and 100\% sensitivity and specificity was determined for the $2 \mathrm{D}$ images (cutoff value, 1.55; Fig 1).

The intraclass correlation coefficient value for the measurements of the plaque CRs was 0.997, indicating excellent intraoperator agreements.

\section{DISCUSSION}

Unstable carotid plaques comprise lipidrich, necrotic, and/or intraplaque hemorrhage materials, enclosed by a thin fibrous cap, which is prone to rupture and subsequently releases embolic materials into the distal blood flow. As a result, unstable plaques cause more ischemic stroke events than stable plaques, which consist mainly of fibrous tissue and/or calcification. ${ }^{12,13}$ Unstable plaques are also thought to be one of the risk factors for embolic complications during carotid endarterectomy and carotid artery stenting. ${ }^{1,2}$ Therefore, accurate characterization of intraplaque components can help predict stroke events and complications during surgery. In this study, we successfully demonstrated that the 3D-FSE T1WI technique can readily discriminate among histologic subtypes of carotid plaques and can differentiate unstable plaques from stable ones, with excellent sensitivity and specificity. These results were comparable with those obtained by using the 2D-SE T1WI technique.

In general, unstable carotid plaques tend to show hyperintensity on T1WI., ${ }^{5,6,14-17}$ However, various imaging techniques have been applied to T1WI, with varying results, including conventional 2D-SE/FSE, ${ }^{5,8}$ cardiac-gated multiple inversion-recovery black-blood 2D-FSE, ${ }^{6}$ MPRAGE, ${ }^{16,18,19}$ and source images of 3D time-of-flight MR angiography. ${ }^{15,20,21} \mathrm{~A}$ recent study performing direct comparisons among these techniques found that 2D-SE imaging could more readily discriminate among fibrous, lipid-rich, necrotic, and hemorrhagic plaques because the $\mathrm{T} 1$ contrast can be maximized and stabilized under the appropriate scanning parameter settings. ${ }^{7}$ In addition, the 2D-SE T1WI could accurately estimate intraplaque composition and could monitor temporal changes during medical treatment. ${ }^{22,23}$ However, the $2 \mathrm{D}$-SE technique is also prone to various technical limitations, such as substantial partial volume effects, susceptibility to motion artifacts, and prolonged acquisition time. The 3D-FSE technique has been introduced recently for plaque imaging and can theoretically overcome these limitations, while still maintaining appropriate $\mathrm{T} 1$ contrast.

In the present study, the 3D-FSE T1WI technique could accurately discriminate among histologically validated plaque types and could also differentiate the vulnerable plaques (types IV-V and VI) from the stable plaques (types VII and VIII) with excellent 
sensitivity and specificity. In particular, the CRs of lipid-rich/necrotic plaques (types IV-V) on 3D-FSE images were significantly higher than those on 2D-SE images obtained with the identical TR. The contrast improvement of the lipid/necrotic plaques by using the $3 \mathrm{D}-\mathrm{FSE}$ technique is thought to be caused by a shortened longitudinal recovery time after the last echo. This augments T1-related contrast, allowing detection of vulnerable plaques that might otherwise have been overlooked. In contrast, the CRs of hemorrhagic plaques (type VI) on 3D-FSE images were significantly lower than those on 2D-SE images, resulting in less accuracy in differentiating hemorrhagic plaques from other plaques, presumably due to the inherent T2 dependency of the 3D-FSE technique caused by its prolonged effective TE. However, this result appears to hardly affect discrimination between unstable and stable plaques because the CRs of hemorrhagic plaques remained remarkably higher than those of stable plaques.

This study includes several limitations. First, although the 3DFSE technique provides potential advantages in terms of partial volume effects, motion artifacts, and multidirectional capability, we did not directly compare these issues between the 2 techniques because our aim was to determine whether 3D-FSE had better contrast for use in plaque characterization than 2D-SE imaging. In general, 3D techniques are suitable for assessment of carotid plaques because the carotid artery is tortuous and pulsatile and the plaques are typically small, complex in shape and composition, and elongated in the superoinferior direction. Hence, 2D imaging is not ideal for visualizing carotid plaque characteristics in detail, given their limited section direction, thickness, and coverage, as well as their susceptibility to motion artifacts. 3D imaging, including 3D-FSE T1WI, might be able to overcome these limitations. Second, we examined CRs of the plaques by using only the section with the maximum plaque size. In this study, we had to abandon the idea of evaluating the whole plaque because only a single section was available for direct comparisons among the $3 \mathrm{D}$ images, $2 \mathrm{D}$ images, and corresponding histologic sections due to partial volume effects of the $2 \mathrm{D}$ images and difficulties in histologic preparation for the appropriate sections. Evaluation of the entire plaque may further improve the diagnostic performance, particularly for the 3D-FSE technique. Third, we could not simultaneously obtain the 3D-FSE and 2D-SE images by using the same scanner because these advanced sequences were implemented in distinct scanners. Hence, the images used for the comparisons may not have been obtained under identical conditions, which might have affected our results, though the intervals between the scans were 2-7 days in almost all patients. Fourth, we did not perform automated analyses that we reported previously, ${ }^{23}$ because appropriate cutoff values for differentiating plaque types on the 3D-FSE images were not known, though they were eventually elucidated in this study.

\section{CONCLUSIONS}

3D-FSE T1WI can accurately characterize carotid plaque composition mainly due to contrast improvement of the lipid-rich plaques and can differentiate vulnerable plaques from stable plaques with excellent sensitivity and specificity, being comparable with 2D-SE T1WI.
Disclosures: Shinsuke Narumi-RELATED: Grant: Grants-in-Aid for Scientific Research (25861122) from the Ministry of Education, Culture, Sports, Science and Technology of Japan. Makoto Sasaki-RELATED: Consulting Fee or Honorarium: Hitachi Medical; UNRELATED: Grants/Grants Pending: Hitachi Medical*; Japanese Ministry Education, Culture, Sports, Science and Technology; Payment for Lectures (including service on Speakers Bureaus): Daiichi Sankyo, Eisai, Sanofi, Tanabe, Johnson \& Johnson, Bayer, AstraZeneca, Kowa, Fuji Film, GE Healthcare. *Money paid to the institution.

\section{REFERENCES}

1. Gaunt ME, Brown L, Hartshorne T, et al. Unstable carotid plaques: preoperative identification and association with intraoperative embolisation detected by transcranial Doppler. Eur J Vasc Endovasc Surg 1996;11:78-82

2. Altaf N, Beech A, Goode SD, et al. Carotid intraplaque hemorrhage detected by magnetic resonance imaging predicts embolization during carotid endarterectomy. J Vasc Surg 2007;46:31-36

3. Waki H, Masuyama T, Mori H, et al. Ultrasonic tissue characterization of the atherosclerotic carotid artery: histological correlates or carotid integrated backscatter. Circ J 2003;67:1013-16

4. Sztajzel R, Momjian S, Momjian-Mayor I, et al. Stratified gray-scale median analysis and color mapping of the carotid plaque: correlation with endarterectomy specimen histology of 28 patients. Stroke 2005;36:741-45

5. Narumi S, Sasaki M, Ohba H, et al. Prediction of carotid plaque characteristics using non-gated MR imaging: correlation with endarterectomy specimens. AJNR Am J Neuroradiol 2013;34:191-97

6. Watanabe $Y$, Nagayama M, Suga T, et al. Characterization of atherosclerotic plaque of carotid arteries with histopathological correlation: vascular wall MR imaging vs. color Doppler ultrasonography(US). J Magn Reson Imaging 2008;28:478-85

7. Saito A, Sasaki M, Ogasawara K, et al. Carotid plaque signal differences among four kinds of $\mathrm{T} 1$-weighted magnetic resonance imaging techniques: a histopathological correlation study. Neuroradiology 2012;54:1187-94

8. Narumi S, Sasaki M, Ohba H, et al. Altered carotid plaque signal among different repetition times on T1-weighted magnetic resonance plaque imaging with self-navigated radial-scan technique. Neuroradiology 2010;52:285-90

9. North American Symptomatic Carotid Endarterectomy Trial Collaborators. Beneficial effect of carotid endarterectomy in symptomatic patients with high-grade carotid stenosis. $N$ Engl $\mathrm{J}$ Med 1991;325:445-53

10. Pipe JG. Motion correction with PROPELLER MRI: application to head motion and free-breathing cardiac imaging. Magn Reson Med 1999;42:963-69

11. Cai JM, Hatsukami TS, Ferguson MS, et al. Classification of human carotid atherosclerotic lesions with in vivo multicontrast magnetic resonance imaging. Circulation 2002;106:1368-73

12. Altaf N, MacSweeney ST, Gladman J, et al. Carotid intraplaque hemorrhage predicts recurrent symptoms in patients with high-grade carotid stenosis. Stroke 2007;38:1633-35

13. Takaya N, Yuan C, Chu B, et al. Association between carotid plaque characteristics and subsequent ischemic cerebrovascular events: a prospective assessment with MRI-initial results. Stroke 2006;37:818-23

14. Cappendijk VC, Cleutjens KB, Kessels AG, et al. Assessment of human atherosclerotic carotid plaque components with multisequence MR imaging: initial experience. Radiology 2005;234:487-92

15. Chu B, Kampschulte A, Ferguson MS, et al. Hemorrhage in the atherosclerotic carotid plaque: a high-resolution MRI study. Stroke 2004;35:1079-84

16. Yamada N, Higashi M, Otsubo R, et al. Association between signal hyperintensity on T1-weighted MR imaging of carotid plaques and ipsilateral ischemic events. AJNR Am J Neuroradiol 2007;28:287-92

17. Yoshida K, Narumi O, Chin M, et al. Characterization of carotid atherosclerosis and detection of soft plaque with use of black-blood MR imaging. AJNR Am J Neuroradiol 2008;29:868-74

18. Moody AR, Murphy RE, Morgan PS, et al. Characterization of compli- 
cated carotid plaque with magnetic resonance direct thrombus imaging in patients with cerebral ischemia. Circulation 2003;107:3047-52

19. Ota H, Yarnykh VL, Ferguson MS, et al. Carotid intraplaque hemorrhage imaging at 3.0-T MR imaging: comparison of the diagnostic performance of three T1-weighted sequences. Radiology 2010;254: $551-63$

20. Yoshimura S, Yamada K, Kawasaki M, et al. High-intensity signal on time-of-flight magnetic resonance angiography indicates carotid plaques at high risk for cerebral embolism during stenting. Stroke 2011;42:3132-37

21. Qiao Y, Etesami M, Malhotra S, et al. Identification of intraplaque hemorrhage on MR angiography images: a comparison of contrastenhanced mask and time-of-flight techniques. AJNR Am J Neuroradiol 2011;32:454-59

22. Yamaguchi M, Sasaki M, Ohba H, et al. Quantitative assessment of changes in carotid plaques during cilostazol administration using three-dimensional ultrasonography and non-gated magnetic resonance plaque imaging. Neuroradiology 2012;54:939-45

23. Narumi S, Sasaki M, Ohba H, et al. Predicting carotid plaque characteristics using quantitative color-coded T1-weighted MR plaque imaging: correlation with carotid endarterectomy specimens. AJNR Am J Neuroradiol 2014;35:766-71 